Original article

https://www.journal-imab-bg.org

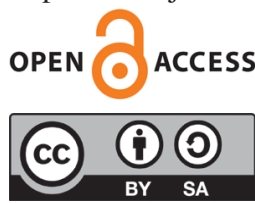

\title{
THE EFFECT OF DIFFERENT IRRIGANTS WITH OR WITHOUT ULTRASONIC ACTIVATION ON ROOT DENTIN MICROHARDNESS
}

\author{
Irina Tsenova-Ilieva, Emilia Karova, \\ Department of Conservative Dentistry, Faculty of Dental Medicine, Medical \\ University - Sofia, Bulgaria.
}

\section{SUMMARY}

Purpose: The aim of this in vitro study was to evaluate the effect of different irrigation regimens of root dentin microhardness.

Materials and methods: Twenty extracted, singlerooted, non-endodontically treated upper incisors were decoronated and further sectioned longitudinally in buccolingual direction. The surfaces of all forty samples were polished, evenly distributed into four groups $(n=10)$ and then immersed in the following solutions: group 1:2\% sodium hypochlorite for 2 minutes followed by $17 \%$ EDTA for 2 minutes; group 2: $2 \%$ sodium hypochlorite for 2 minutes followed by $17 \%$ EDTA for 2 minutes, both ultrasonically activated; group 3: 5.25\% sodium hypochlorite for 2 minutes followed by $17 \%$ EDTA for 2 minutes; group 4 : distilled water (control group). Afterwards, the samples were dried and subjected to microhardness testing by using $300 \mathrm{~g}$ load with a dwell time of 20 seconds. The averaged values of each sample's Vicker's hardness numbers were calculated and statistically analyzed.

Results: All irrigation regimens significantly decreased the mean hardness of dentin when compared to the control group $4(\mathrm{p}<0.05)$. Pair-wise comparison between the values of Group 1, Group 2, Group 3 resulted in no statistical significance $(p>0.05)$ regardless of the concentration and the ultrasonic activation of the solutions.

Conclusions: The dentin microhardness was decreased regardless of the irrigation protocols used. Additional studies should clarify the clinical relevance of the compromised mechanical properties of dentin tissue and their relationship with the overall treatment success.

Keywords: Dentin microhardness, irrigation, sodium hypochlorite, EDTA,

\section{INTRODUCTION}

The main goal of the chemo-mechanical debridement in the course of the endodontic treatment is to eradicate microbiota from the root canal system and ensure an adequate three-dimensional, tight-seal filling of the root canal space, thus increasing the long-term survival of nonvital teeth [1]. Mechanical instrumentation using either manual or rotary files produces a thin, loosely-bonded, amorphous smear layer composed of organic and inorganic substances such as pulp tissue debris, odontoblastic processes, dentin particles, microorganisms and their metabolic products [2, 3]. Ideally, the irrigants should provide lubrication, debridement, antimicrobial effect and remove the smear layer entirely. However, there is no single irrigant solution that can fulfil these requirements [4].

Reports concerning the retention of the smear layer as a barrier against bacteria and other irritants are still inconsistent $[4,5]$. During the smear layer removal, irrigation solutions cause alterations in the chemical composition of dentin. The change of $\mathrm{Ca} / \mathrm{P}$ ratio affects the original proportion of organic to inorganic components, which in turn changes the microhardness, solubility, permeability and surface roughness of dentin. These effects depend on the application time, the concentration of the irrigants and the irrigation protocol used throughout the endodontic treatment [1].

The most commonly used endodontic irrigant is $0.5 \%$ to $6.25 \%$ sodium hypochlorite solution $(\mathrm{NaOCl})$. It is an antibacterial agent capable of dissolving vital and necrotic organic tissue, as well as the organic component of the smear layer. Sodium hypochlorite fragments long peptide chains and chlorinates protein terminal groups, resulting in $\mathrm{N}$-chloramines that are further broken down into other species. The degradation of the organic components by $\mathrm{NaOCl}$ solutions can alter adversely dentinal biomechanics by significantly decreasing its elastic modulus and flexural strength $[1,3]$.

Despite its advantages, $\mathrm{NaOCl}$ is insufficient for achieving thorough cleaning and disinfection of the root canal when used alone $[1,3]$. Thus, successive rinses with chelating agents and $\mathrm{NaOCl}$ solutions have been advocated as an irrigation regimen for efficient smear layer removal $[6,7,8]$. Chelators are complexions capable of binding single calcium ions present in the hydroxyapatite crystals of dentin. This results in softening of the root canal wall, which facilitates the instrumentation process and prepares the dentin for better adhesion of filling materials [1].

The most frequently used chelators are based on different concentrations of ethylenediaminetetraacetic acid (EDTA) [1,9]. The slightly alkaline $(\mathrm{pH}=7.7)$ disodium salt of $17 \%$ EDTA chelates and removes the mineralized ingredients of the smear layer $[3,8]$. The demineralization process is self-limited since EDTA loses its activity in cases of 
complete saturation of chelator complexes with calcium ions [1].

Most of the studies compare the microhardness change after the single use of an endodontic irrigant. However, a few of them focus on the combined use of the most common disinfection solutions. There is a lack of consensus in the literature regarding the optimal application time and concentration of the disinfection solutions. To the best of our knowledge, there are no articles that investigate the effect of ultrasonically activated successive rinses with $\mathrm{NaOCl}$ and EDTA on root dentin microhardness. The aim of the current study is to assess the effect of three different irrigation regimens with or without ultrasonic activation on the microhardness of root canal dentin. The null hypothesis is that there will be no reduction of the root dentin microhardness after application of different irrigation protocols.

\section{MATERIALS AND METHODS}

\section{Sample selection and preparation}

Approval of the study was obtained by the Research Ethics Committee of Medical University - Sofia, Sofia, Bulgaria (1838/05.03.2020). All samples in the experiment were recruited from a pool of recently extracted due to periodontal lesions upper incisors from the Department of Oral and Maxillofacial Surgery, Faculty of Dental Medicine, Medical University - Sofia, Bulgaria. All patients signed informed consent. Overall, twenty teeth from patients aged 40-55 years were obtained and stored in distilled water until further use. The external surface of all samples was cleaned from calculus and plaque using periodontal $\mathrm{cu}$ rettes. Each tooth was radiographed to confirm the presence of a single canal. Teeth were selected based on their relative dimensions and morphological similarities. Afterwards, the teeth were examined under a stereomicroscope Leica S6 (Leica Microsystems, Wetzlar, Germany) at x20 and x40 magnification for detection of external root damage. All incisors presenting any root or coronal defects such as caries and cracks, apical curvature greater than $5^{\circ}$, fractured and/or immature root apices, calcifications, internal or external resorption were excluded from the investigation and replaced with new ones. No endodontic treatment was initiated for the samples. All teeth were decoronated at the cementoenamel junction by using diamond disk under water cooling leaving the roots at $16 \mathrm{~mm}$ length. The roots were further sectioned longitudinally in the buccolingual direction by saw microtome Leica SP1600 (Leica Microsystems, Wetzlar, Germany) under copious water cooling, and a total number of 40 samples was obtained. Root specimens were horizontally embedded into acrylic resin moulds, leaving the dentinal surface exposed. The surface of each root half was polished with polishing discs OptiDisc (Kerr Dental, Orange, CA, USA) in the following order: ExtraCoarse $(80 \mu \mathrm{m})$, Coarse/Medium $(40 \mu \mathrm{m})$, Fine $(20 \mu \mathrm{m})$, Extra-Fine $(10 \mu \mathrm{m})$ under copious water coolant and the integrity of the dentinal surface was reevaluated stereomicroscopically. The prepared samples were evenly distributed into four experimental groups $(n=10)$ and immersed in the irrigant solutions as follows:
Group 1: Samples were immersed in $50 \mathrm{ml} 2 \%$ sodium hypochlorite $(\mathrm{NaOCl})$ for 2 minutes then in $50 \mathrm{ml} 17 \%$ EDTA for 2 minutes.

Group 2: Samples were immersed in $50 \mathrm{ml} \mathrm{2 \%} \mathrm{so-}$ dium hypochlorite $(\mathrm{NaOCl})$ for 2 minutes then in $50 \mathrm{ml} 17 \%$ EDTA for 2 minutes (both solutions were ultrasonically activated for $2 \mathrm{~min}$ ).

Group 3: Samples were immersed in $50 \mathrm{ml} 5.25 \%$ sodium hypochlorite $(\mathrm{NaOCl})$ for 2 minutes then in $50 \mathrm{ml}$ 17\% EDTA for 2 minutes.

Group 4: Samples were immersed in $5 \mathrm{ml}$ distilled water (control group).

The ultrasonic agitation in group 2 was obtained by using a Digital Ultrasonic Cleaner CD-4820 (Shenzhen Codyson Electrical Co., Ltd, China) at 42,000 Hz frequency. Immediately after the action of each of the irrigant solutions, all specimens were rinsed with $50 \mathrm{ml}$ distilled water to eliminate the chemical interaction between them and the prolonged effect of the chelating agent. Afterwards, the samples were left to dry on absorbent paper and then mounted on the stage of Micro Vickers \& Knoop Hardness Tester (Indentec Hardness Testing Machines Limited, United Kingdom). Each root half was divided into three segments - coronal, middle and apical. Two indentations were made on the dentinal surface on both sides of the canal lumen in the three tested areas of each sample. Each measurement was carried out using a $300 \mathrm{~g}$ load with a dwell time of 20 seconds, applied at approximately $500 \mu \mathrm{m}$ distance from the pulp-dentin interface, oriented perpendicularly to the root surface. The diagonal lengths of the indentations were calculated by built in software and automatically converted into Vickers hardness numbers (VHN) displayed on the monitor of the device. All six values were averaged to produce the final microhardness value of each specimen.

\section{STATISTICAL ANALYSIS}

Data were tabulated for statistical analysis using the SPSS software (SPSS v.25.0.0.0, SPSS INC, IL, USA). Statistical analysis for intergroup comparison was carried out using one-way ANOVA. Post-hoc Tuckey test was performed to find out the pair-wise mean microhardness difference between two groups. The confidence level was set at $95 \%(\mathrm{p}<0.05)$.

\section{RESULTS}

Vickers microhardness values (mean \pm S.D.) for the irrigating protocols are summarized in table 1. One-way ANOVA demonstrated that there was a statistically significant difference in the mean hardness among the four groups after the specimens were immersed in their respective irrigants $(\mathrm{p}<0.05)$ (Table 2). The subsequent post-hoc Tuckey test showed that the three experimental groups reduced the mean hardness of dentin significantly when compared to the control group $4(\mathrm{p}<0.05)$. Pair-wise comparison between the values of Group 1, Group 2, Group 3 resulted in no statistical significance $(\mathrm{p}>0.05)$ regardless of the concentration and the ultrasonic activation of the solutions (Table 3). 
Table 1. Descriptive statistic of microhardness values for all experimental groups.

\begin{tabular}{|l|c|c|c|c|c|}
\hline Groups & $\boldsymbol{N}$ & Mean & \pm S.D. & Min & Max \\
\hline Group 1 & 10 & 51.58 & \pm 1.25 & 49.33 & 52.67 \\
\hline Group 2 & 10 & 50.38 & \pm 1.05 & 48.67 & 51.67 \\
\hline Group 3 & 10 & 50.41 & \pm 1.46 & 48.67 & 52.33 \\
\hline Group 4 (control) & 10 & 58.18 & \pm 1.33 & 56.33 & 60.00 \\
\hline
\end{tabular}

Table 2. One-way ANOVA test of the mean values of all tested groups.

\begin{tabular}{|l|c|c|c|c|c|}
\hline & Sum of Squares & df & Mean Square & F & $\boldsymbol{p}$-value \\
\hline Between groups & 418.89 & 3 & 139.62 & 84.82 & .000 \\
\hline Within groups & 59.26 & 36 & 1.65 & & \\
\hline Total & 478.15 & 39 & & & \\
\hline
\end{tabular}

Table 3. Pair-wise multiple comparisons of microhardness between groups using post-hoc Tuckey test.

\begin{tabular}{|c|c|c|}
\hline Pairs & p-value & Significance \\
\hline Group 1 - Group 2 & 0.178 & Not significant \\
\hline Group 1 - Group 3 & 0.193 & Not significant \\
\hline Group 1 - Group 4 & 0.000 & Significant \\
\hline Group 2 - Group 3 & 1.000 & Not significant \\
\hline Group 2 - Group 4 & 0.000 & Significant \\
\hline Group 3 - Group 4 & 0.000 & Significant \\
\hline
\end{tabular}

\section{DISCUSSION}

All of the tested irrigation regimens in the current experiment significantly reduced the microhardness of the root dentin compared to the control group, thus the null hypothesis was rejected. Our results confirmed that disinfection solutions used during cleaning and shaping of the root canal might cause alterations of the mechanical properties of radicular dentin, such as its hardness $[3,5,10]$.

Microhardness testing is one of the most widely used, non-destructive and simple methods to investigate fine scale changes in the hardness of dentin structure. It is defined as the resistance to local deformation measured on the basis of the induced permanent surface deformation that remains after removal of the load [11]. Although this in vitro test is not clinically relevant, the determination of the hardness profile can provide indirect evidence of mineral loss or gain in dental hard tissues [12]. Such changes may affect the adhesive properties of root canal dentin and decrease the root strength, thus increasing its fracture susceptibility [13].

The accurate readings of Vickers hardness test and the usage of just one type of indentation for all surface treatments make it useful for registration of surface changes of dental hard tissues treated with chemical agents explored by us [14]. In an attempt to preserve the integrity of the root canal wall by unnecessary removal of root canal den- tin and provide a plane for measurement as close as the original morphology of the dentinal surface, the specimens were not mechanically prepared $[5,10,15]$. In the current experiment, the longitudinal sectioning of the teeth was preferred over the transverse cutting into discs as CruzFilho et al. observed that it can show a more accurate representation of clinical situations $[4,8,13]$.

Biological materials, such as dentin, are less homogenous, and this may cause discrepancies in the results because of differences in the structure of adjacent regions of root dentin $[4,16]$. Despite being structurally different, the dentin surface in the root thirds behaves similarly in terms of microhardness change when subjected to one and the same irrigation regimen [17]. Based on this assumption, we calculated the mean hardness value of the coronal, middle and apical third per each sample. Pashley et al. (1985) stated that dentin hardness is location-related, and its value decreases as the indentations were made close to the pulp [18]. This phenomenon is explained with the increased number of widely-open dentinal tubules with a decreased amount of intertubular dentin near the pulp, causing little resistance to the testing indenter [19]. In an attempt to standardize the circumstances for all experimental groups and allow for comparable results, the testing was performed at $500 \mu \mathrm{m}$ distance from the canal lumen of each root half $[9,11,12]$. The application of $300 \mathrm{~g}$ load for $20 \mathrm{sec}$ dwell- 
ing time was consistent with previous microhardness studies $[4,5]$.

In order to guarantee unbiased evaluation, we used a control group, where all specimens were immersed in distilled water $[5,9,10,11,17]$. It was used as a storage media, interim irrigant and immersion solution in the control group because it has no effect on dentin surface, and thus not considered as a variable which might affect the results [3].

There is a lack of consensus in the research data on the application time and concentration of the endodontic irrigants for achieving an optimal smear layer removal without impairing the mechanical properties of root dentin. A lot of previous investigations showed that the use of sodium hypochlorite alone leads to the reduction of dentin microhardness and this effect is concentration-dependent $[1,5]$. Slutzky-Goldberg et al. (2002) concluded that, at a depth of $500 \mu \mathrm{m}$ from the lumen, the decrease of microhardness is more pronounced after exposure of dentin to $6 \% \mathrm{NaOCl}$ than to $2.5 \% \mathrm{NaOCl}$ and this statement was confirmed by a recent study of Duvvi et al. (2018) [14, 4]. Numerous studies reported that the increased exposure time of different concentrations of chelating agents might lower dentin microhardness in greater extent $[1,16]$. Calt and Serper (2002) observed that reduction of dentin hardness after the 5-minute action of the chelating agent was substantially higher than its 1-minute application at a depth of $100 \mu \mathrm{m}$ from the canal lumen [7].

In an attempt to resemble the clinical situation, various authors advocate the 5-minute duration of the endodontic solutions $[13,16]$. Additionally, De-Deus et al. evaluated the effect of the single use of $17 \%$ EDTA for periods of 1, 3 and 5 minutes on the microhardness of root dentin. Their findings showed that EDTA produced the greatest decrease in microhardness from reference state to $3 \mathrm{~min}$ utes with no further progression after a 5 -minute period [8, 16]. The immersion time in each irrigant in our experiment was 2 minutes. The results we obtained demonstrated that even shorter duration of various combinations and concentrations of endodontic irrigants with or without ultrasonic agitation significantly reduced the root dentin microhardness compared to the untreated control group. Our results corroborate with the findings of Akcay et al. who stated that after a one-minute application time of $7.5 \%$ EDTA followed by $2.5 \% \mathrm{NaOCl}$ the dentin microhardness is lower than its reference pre-treatment value [15].

Niu et al. demonstrated dentin erosion occurs when $\mathrm{NaOCl}$ is applied after EDTA. The demineralized dentinal tubules characterize with enlarged orifice diameters and reduced intertubular dentin thickness. This, in turn, might cause changes in the viscoelastic properties of dentin [6]. In order to prevent further erosion, we immersed the specimens $\mathrm{NaOCl}$ solution only once, before the application of EDTA [5]. Sodium hypochlorite is an efficient organic tissue solvent that causes dissolution of collagen by the breakdown of the bonds between carbon atoms and disorganization of the protein's primarily structure and change in magnesium and phosphate ions [8]. Thus, an apatite-rich, collagen-spares dentin subsurface is formed that is more brittle than untreated mineralized dentin. This subsurface zone has the potential to create non-uniform deproteinization channels that favor subsequent EDTA penetration and further apatite dissolution [5]. Demineralization of root dentin with consequent exposure of collagen leads to decreased dentin microhardness as observed in the present research. Our results are consistent with those obtained by Hanaa Saleh and other authors investigating the irrigation protocols with the initial application of EDTA followed by $\mathrm{NaOCl}[5,8,15]$.

It has been stated that the use of endodontic solutions in conjunction with ultrasonic agitation, generating a continuous movement of the irrigant, is directly associated with the effectiveness of the cleaning of the root canal [20]. It might be speculated that the additional activation of the disinfection irrigants can result in a higher decrease of dentin microhardness compared to that of the nonactivated groups using the same or higher concentrations of $\mathrm{NaOCl}$. Nonetheless, the decrease in the microhardness value among the three experimental groups remained statistically insignificant. This might be due to the short duration period and the methodology utilized for the ultrasonic activation, which differs from the clinical condition of the passive ultrasonic activation.

A possible limitation of the present study is that the experimental conditions differ substantially from the clinical situation. The immersion treatment is performed with a greater volume of the irrigants compared with the amount used in a closed root canal. Clinically, the root canal space is a closed-end channel that can generate vapour-lock effect during irrigation. Hence, different parts of the root canal wall are affected differently by disinfection solutions [4]. The penetration of irrigants is lower in the apical third of the canal, and little amount of them is in contact with the root canal walls at that level [8]. Thus, it might be expected that the deleterious effect of the irrigation protocols on the mechanical properties of the coronal and middle dentin will be greater. However, the immersing methodology guarantees a uniform application of a relatively large amount of the irrigant so that it remains in close contact with the entire dentine surface. Further studies should evaluate the effect of various irrigant regimens in a closedcanal system in conjunction with or without ultrasonic agitation systems [4].

\section{CONCLUSIONS}

Within the limitations of the current study, it could be concluded that:

1) All tested irrigants significantly decreased the microhardness of root dentin.

2) The effect of different irrigation protocols on the dentinal surface was similar and remained statistically insignificant.

\section{ACKNOWLEDGMENTS}

This article is sponsored by the Scientific Council of Medical University - Sofia, Bulgaria, Grant Project No. 8326/22. 11. 2018; Contract No. 90/23. 04. 2019. 


\section{REFERENCES:}

1. Tsenova I, Vassileva R, Karova E. The ability of root canal cleaning and shaping procedures to initiate dentinal radicular microcracks. Int $J$ Sci Res. 2018 Aug;7(8):1558-1565.

2. Rapgay T, Gupta P, Gupta H, Jain A. Effect of different irrigant solutions on root dentine microhardness: an in vitro study. Acta Scientific Dent Sci. 2018 Aug;2(8):3-8.

3. Kandil HE, Labib Ah, Alhadainy HA. Effect of different irrigant solutions on microhardness and smear layer removal of root canal dentin. Tanta Dent J. 2004 Apr;11(1):1-11. [Crossref]

4. Duvvi SAB, Adarsha MS, Usha HL, Ashwini P, Murthy CS, Shivekshith AK. Comparative assessment of different concentrations of sodium hypochlorite and calcium hypochlorite on microhardness of root canal dentin - an in vitro study. Int J Oral Care Res. 2018 Jan-Mar;6(1):54-58.

5. Saleh HAJ. Comparative evaluation of effect of irrigation solutions with various exposure time on microhardness of root canal dentin (in vitro study). Iraqi Dent J. 2016 Dec;38(3): 124-128. [Crossref]

6. Niu W, Yoshioka T, Kobayashi C, Suda H. A scanning electron microscopic study of dentinal erosion by final irrigation with EDTA and $\mathrm{NaOCl}$. Int Endod J. 2002 Nov;35(11):934-9. [PubMed]

7. Calt S, Serper A. Time-dependent effects of EDTA on dentin structures. J Endod. 2002 Jan;28(1):17-9.
[PubMed]

8. Massoud SF, Moussa SM, Hanafy SA, El Backly RM. Evaluation of the microhardness of root canal dentin after different irrigation protocols (in vitro study). Alex Dent J. 2017 Spring; 42(1):73-79. [Crossref]

9. Baldasso FER, Roleto L, Silva VDD, Morgental RD, Kopper PMP. Effect of final irrigation protocols on microhardness reduction and erosion of root canal dentin. Braz Oral Res. 2017 May 15;31:e40:1-8. [PubMed]

10. Quiteifan MQ, Madarati AA, Layous K, Al Tayyan M. A comparative ex-vivo study of effects of different irrigation protocols with/without laser activation on the root dentine's micro-hardness. Eur Endod J. 2019 Dec 3;4(3):127-132. [PubMed]

11. Abbas FS, Abdul NJ, Hassan AS. Effect of final irrigation protocols on dentin microhardness. Biomed Pharm J. 2018 Dec;11(4):2157-2162. [Crossref]

12. Das A, Kottoor J, Mathew J, Kumar S, George S. Dentin microhardness changes following conventional and alternate irrigation regimens: an in vitro study. J Conserv Dent. 2014 Nov-Dec;17(6):546-9. [PubMed]

13. Cruz-Filho AM, Souza-Netto Md, Savioli RN. Effect of chelating solutions on the microhardness of root canal lumen dentin. J Endod. 2011 Mar;37(3):358-62. [PubMed]

14. Slutzky-Goldberg I, Maree M, Liberman R, Heling I. Effect of sodium hypochlorite on dentin microhardness.
J Endod. 2002 Dec;30(12):880-2. [PubMed]

15. Akcay I, Erdilek N, Sen BH. The efficacy of experimental single solution versus alternate use of multiple irrigants on root dentin microhardness. J Clin Exp Dent. 2013 Apr 1;5(2):e83-8. [PubMed]

16. De-Deus G, Paciornik S, Mauricio MHP. Evaluation of the effect of EDTA, EDTAC and citric acid on the microhardness of root dentine. Int Endod J. 2006 May;39(5):401-7. [PubMed]

17. Tartari T, de Almeida Rodrigues Silva E Souza P, Vila Nova de Almeida B, Carrera Silva Júnior JO, Facíola Pessoa O, Silva E Souza Junior MH. A new weak chelator in endodontics: effects of different irrigation regimens with Etidronate on root dentin microhardness. Int J Dent. 2013; 2013: 743018. [PubMed]

18. Pashley DA, Parham P. The relationship between dentin microhardness and tubule density. Endod Dent Traumatol. 1985 Oct;1(5):176-9. [PubMed]

19. Kinney JH, Marshall SJ, Marshall GW. The mechanical properties of human dentin: a critical review and re-evaluation of the dental literature. Crit Rev Oral Biol Med. 2003 Jan; 14(1):13-29. [PubMed]

20. Plotino G, Pameijer CH, Grande NM, Somma F. Ultrasonics in endodontics: a review of the literature. $J$ Endod. 2007 Feb;33(2):81-95. [PubMed]

Please cite this article as: Tsenova-Ilieva I, Karova E. The effect of different irrigants with or without ultrasonic activation on root dentin microhardness. J of IMAB. 2021 Jan-Mar;27(1):3534-3538.

DOI: https://doi.org/10.5272/jimab.2021271.3534

Received: 20/03/2020; Published online: 19/01/2021

\author{
Address for correspondence: \\ Irina Tsenova-Ilieva \\ Assistant Professor, Department of Conservative Dentistry, Faculty of Dental \\ Medicine, Medical University - Sofia, \\ 1, St Georgi Sofiyski blvd., 1413 Sofia, Bulgaria \\ E-mail: irinatsenova@yahoo.com
}

